\title{
On distance and Laplacian matrices of trees with matrix weights
}

\author{
Fouzul Atik* $\quad$ M. Rajesh Kannan ${ }^{\dagger} \quad$ R. B. Bapat ${ }^{\ddagger}$
}

June 26, 2021

\begin{abstract}
The distance matrix of a simple connected graph $G$ is $D(G)=\left(d_{i j}\right)$, where $d_{i j}$ is the distance between the vertices $i$ and $j$ in $G$. We consider a weighted tree $T$ on $n$ vertices with edge weights are square matrix of same size. The distance $d_{i j}$ between the vertices $i$ and $j$ is the sum of the weight matrices of the edges in the unique path from $i$ to $j$. In this article we establish a characterization for the trees in terms of rank of (matrix) weighted Laplacian matrix associated with it. Then we establish a necessary and sufficient condition for the distance matrix $D$, with matrix weights, to be invertible and the formula for the inverse of $D$, if it exists. Also we study some of the properties of the distance matrices of matrix weighted trees in connection with the Laplacian matrices, g-inverses and eigenvalues.
\end{abstract}

AMS Subject Classification(2010): 05C50, 05C22.

Keywords. Tree, Distance matrix, Laplacian matrix, Matrix weight, Inverse, g-inverse.

\section{Introduction}

Consider an $n$-vertex connected graph $G=(V, E)$, where $V=\{1,2, \ldots, n\}$ is the vertex set and $E=\left\{e_{1}, e_{2}, \ldots, e_{m}\right\}$ is the edge set. The distance matrix $D(G)$ of $G$ is an $n \times n$ matrix $\left(d_{i j}\right)$ such that $d_{i j}$ is the distance (length of a shortest path) between the vertices $i$ and $j$ in $G$. Let $T$ be a tree with $n$ vertices and let $W_{i}$ be the $s \times s$ edge weight matrix associated with the edge $e_{i}, i=1,2, \ldots, n-1$. If $i$ and $j$ are vertices of $T$, then there is a unique path from $i$ to $j$ in $T$. The distance $d_{i j}$ between the vertices $i$ and $j$ is defined to be the sum of the weights matrices of the edges in the unique path connecting them. Then the distance matrix $D$ of $T$ is a block matrix of order $n s \times n s$. Usually the Laplacian matrix of the weighted tree $T$ with matrix weight is the block matrix $\left(l_{i j}\right)$, where $l_{i j}$ is equal to the sum of the weight matrices of the edges incident on vertex $i$ if $i=j$, equal to $-W$ if there is an edge between $i$ and $j$ and weight of that edge is $W$, and zero matrix otherwise. In this article, we consider the Laplacian matrix obtained by replacing each edge weight matrix of the tree by its inverse.

The distance matrix of graphs, in particular trees, have been studied by researchers for many years. An early remarkable result of Graham and Pollak [7] states that, if $T$ is a tree on $n$ vertices,

${ }^{*}$ Theoretical Statistics and Mathematics Unit, Indian Statistical Institute, New Delhi 110 016, India. Email: fouzulatik@gmail.com

${ }^{\dagger}$ Department of Mathematics, Indian Institute of Technology Kharagpur, Kharagpur, India. Email: rajeshkannan@maths.iitkgp.ernet.in, rajeshkannan1.m@gmail.com

${ }^{\ddagger}$ Theoretical Statistics and Mathematics Unit, Indian Statistical Institute, New Delhi 110 016, India. Email: rbb@isid.ac.in 
then the determinant of the distance matrix $D$ of $T$ is $(-1)^{n-1}(n-1) 2^{n-2}$, which is independent of the structure of the underlying tree. Subsequently, Graham and Lovász [6] derived a formula for the inverse of the distance matrix of a tree. An extension of these two results for the weighted trees, where the weights are being positive scalars, were obtained by Bapat et al. [2]. In [3], Bapat obtained the determinant of the distance matrix of a weighted tree where weights are arbitrary matrices of fixed order. In [1], Balaji and Bapat determined the inverse of the distance matrix of a weighted tree where weights are positive definite matrices. Bapat et al., considered the $q$-distance matrix of an unweighted tree and gave formulae for the inverse and the determinant [4]. Similar type of results were established for distance matrices of trees with weights are from rings [10, 11].

In this paper we consider weighted trees with the edge weights are matrices of same size. We establish a characterization for trees in terms of rank of (matrix) weighted Laplacian matrix associated with it(Theorem 2.4). Then we derive a necessary and sufficient condition for the distance matrix to be invertible and the formula for the inverse of the distance matrix, if it exist(Theorem [3.2). Also we study some of the properties of the distance matrices of matrix weighted trees in connection with the Laplacian matrices(Theorem 3.3) and g-inverses(Theorem 3.4). Finally, we derive an interlacing inequality for the eigenvalues of distance and Laplacian matrices for the case of positive definite matrix weights(Theorem 3.6).

This article is organized as follows: In section 2, we study some of the properties of the matrix weighted Laplacian matrices of graphs and provide a characterization for trees in terms rank of weighted Laplacian matrix associated with it. In section 3, we establish a formula for the inverse of distance matrix of weighted trees whose weights are invertible matrices. Using this, we prove some properties of distance matrices related to Laplacian matrices, g-inverse and eigenvalues.

\section{A characterization for trees}

In this section, first we define the notion of incidence matrix of a weighted graph whose edges are assigned positive definite matrix weights.

Definition 2.1. Let $G$ be a connected weighted graph with $n$ vertices and $m$ edges and all the edge weights of $G$ are positive definite matrices of order $s \times s$. We assign an orientation to each edge of $G$. Then the vertex edge incidence matrix $Q$ of $W$ is the $n s \times$ ms block matrix whose row and column blocks are index by the vertices and edges of $G$, respectively. The $(i, j)$ th block of $Q$ is zero matrix if the ith vertex and the $j$ th edge are not incident and it is ${\sqrt{W_{j}}}^{-1}$ (respectively, $-{\sqrt{W_{j}}}^{-1}$ ) if the ith vertex and the $j$ th edge are incident, and the edge originates (respectively, terminates) at the ith vertex, where $W_{j}$ denotes the weight of the $j$ th edge.

In this case, it is easy to see that, the Laplacian matrix $L$ can be written as $L=Q Q^{T}$.

Recall that, the Kronecker product of matrices $A=\left(a_{i j}\right)$ of size $m \times n$ and $B$ of size $p \times q$, denoted by $A \otimes B$, is defined to be the $m p \times n q$ block matrix $\left(a_{i j} B\right)$. It is known that for matrices $M, N, P$ 
and $Q$ of suitable sizes, $M N \otimes P Q=(M \otimes P)(N \otimes Q)[8$.

Theorem 2.1. If $G$ is a connected weighted graph on $n$ vertices such that the edge weights are $s \times s$ positive definite matrices, then rank of $Q$ as well as rank of $L$ is $(n-1) s$.

Proof. We have $Q^{T}$ is a matrix of order $m s \times n s$, where $m$ is the number of edges in $G$. Let $X$ is a vector in the null space in $Q^{T}$ and let $X=\left(X_{1}, X_{2}, \cdots, X_{n}\right)^{T}$ be a partition where each $X_{i}$ is of order $1 \times s$. Then $Q^{T} X=0$ gives $\left(\sqrt{W_{i}}\right)^{-1}\left(X_{i}-X_{j}\right)=0$, where $W_{i}$ is the weight of the $(i, j)$ th edge in $G$. Since $W_{i}$ is positive definite, $X_{i}=X_{j}$. As the graph $G$ is connected, we must have $X_{i}=X_{j}$ for all $i, j=1,2, \cdots, n$. Thus $X$ must be of the form $e^{T} \otimes X_{1}^{T}$, where $e$ is a all one vector of order $n$. So the dimension of null space of $Q^{T}$ is at most $s$ and thus rank of $Q^{T}$ is at least $(n-1) s$. Again the block row sum of $Q^{T}$ is zero, gives rank of $Q^{T}$ is at most $(n-1) s$. Hence rank of $Q^{T}$ and $Q$ is $(n-1) s$. Also as the edge weights of the graphs are positive definite matrices, so $L=Q Q^{T}$ and rank of $L$ is $(n-1) s$.

Next we give an example of a weighted graph (not a tree) with weights are nonsingular matrix for which rank of $L$ is not $(n-1) s$.

Example 2.1. Consider the cycle of order 4 (Figure 1), where the edge weights are $W_{1}=\left[\begin{array}{ll}1 & 0 \\ 0 & 1\end{array}\right], W_{2}=$ $\left[\begin{array}{ll}0 & 1 \\ 1 & 0\end{array}\right], W_{3}=\left[\begin{array}{ll}1 & 0 \\ 0 & 1\end{array}\right]$, and $W_{4}=\left[\begin{array}{ll}0 & 1 \\ 1 & 0\end{array}\right]$ and then the Laplacian matrix is given by

$$
\begin{aligned}
L & =\left[\begin{array}{cccccc}
W_{1}^{-1}+W_{4}^{-1} & -W_{1}^{-1} & 0 & -W_{4}^{-1} \\
-W_{1}^{-1} & W_{1}^{-1}+W_{2}^{-1} & -W_{2}^{-1} & 0 \\
0 & \multicolumn{2}{c}{-W_{2}^{-1}} & W_{2}^{-1}+W_{3}^{-1} & -W_{3}^{-1} \\
-W_{4}^{-1} & & 0 & & -W_{3}^{-1} & W_{3}^{-1}+W_{4}^{-1}
\end{array}\right] \\
& =\left[\begin{array}{cccccccc}
1 & 1 & -1 & 0 & 0 & 0 & 0 & -1 \\
1 & 1 & 0 & -1 & 0 & 0 & -1 & 0 \\
-1 & 0 & 1 & 1 & 0 & -1 & 0 & 0 \\
0 & -1 & 1 & 1 & -1 & 0 & 0 & 0 \\
0 & 0 & 0 & -1 & 1 & 1 & -1 & 0 \\
0 & 0 & -1 & 0 & 1 & 1 & 0 & -1 \\
0 & -1 & 0 & 0 & -1 & 0 & 1 & 1 \\
-1 & 0 & 0 & 0 & 0 & -1 & 1 & 1
\end{array}\right]
\end{aligned}
$$

Then one can verify that rank of $L$ is $5(\neq(n-1) s)$. 


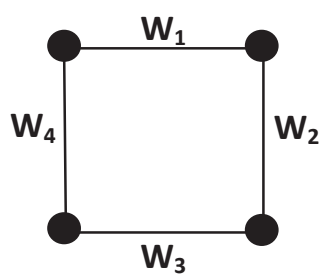

Figure 1: Cycle of order 4

From the previous example we can see that for a weighted graph (not a tree) with weights are nonsingular matrices for which rank of $L$ may not be $(n-1) s$. However from the theorem below, it is guaranteed that if the graph is tree then for nonsingular matrix weights of order $s$, then the rank of $L$ is $(n-1) s$.

Theorem 2.2. If $T$ is a weighted tree on $n$ vertices such that the edge weights are $s \times s$ nonsingular matrices, then rank of $L$ is $(n-1) s$.

Proof. To prove the result we use induction on the number of vertices $n$ of the tree $T$. For $n=2, T$ is just an edge and let weight on this edge is $W$, which is a nonsingular matrix of order $s$. Then clearly $\operatorname{rank}$ of $L=\left[\begin{array}{cc}W_{1}^{-1} & -W_{1}^{-1} \\ -W_{1}^{-1} & W_{1}^{-1}\end{array}\right]$ is $s$.

Assume that the result is true for $n=k-1$. Let $T$ be a tree on $k$ vertices. Consider a pendent vertex $v_{1}$ and the vertex $v_{2}$ which is adjacent to $v_{1}$ and let weight of the edge $\left(v_{1}, v_{2}\right)$ is $W$. Let $L$ be the Laplacian matrix of $T$ in which vertex ordering is $v_{1}, v_{2}$ and followed by the other vertices of $T$. Let $T^{*}$ be the tree obtained by removing the vertex $v_{1}$ from $T$ and $L^{*}$ be the corresponding Laplacian matrix of $T^{*}$. Then

$$
L=\left[\begin{array}{cc|ccc}
W^{-1} & -W^{-1} & 0 & \cdots & 0 \\
-W^{-1} & W^{-1}+A & * & \cdots & * \\
0 & * & * & \cdots & * \\
\cdots & \cdots & \cdots & \cdots & \cdots \\
0 & * & * & \cdots & *
\end{array}\right]=\left[\begin{array}{cc|c}
W^{-1} & -W^{-1} 00 \cdots & 0 \\
\hline-W^{-1} & \\
0 & e_{1} e_{1}^{T} \otimes W^{-1}+L^{*} \\
\cdots & \\
0 &
\end{array}\right]
$$

where $A$ is the first diagonal block of the matrix $L^{*}$. Now by induction hypothesis rank of $L^{*}$ is $(n-2) s$. As the block row sum of $L^{*}$ is zero, so we have the last $(n-2) s$ columns of $L^{*}$ are linearly independent. Then last $(n-2) s$ columns of $L$ are also linearly independent. Again as $W$ is nonsingular, so all the $s$ linearly independent columns of $L$ corresponding to the vertex $v_{2}$ is linearly independent with the last $(n-2) s$ columns of $L$. Hence we get $L$ has $(n-1) s$ number of linearly independent columns so that the rank of $L$ is $(n-1) s$.

In the following theorem, we prove that for a graph which is not a tree, there exist an assignment of nonsingular matrix weights for its edges such that rank of $L$ is less than $(n-1) s$. 
Theorem 2.3. Let $G$ be a connected weighted graph on $n$ vertices, which is not a tree. Then there exist an assignment of $s \times s$ nonsingular matrix weights on the the edges of $G$ such that rank of $L$ is less than $(n-1)$ s.

Proof. To prove the result, it is sufficient to give an assignment of non zero scaler weights on the edges of the graph $G$ such that rank of $L$ is less than $(n-1)$. As $G$ is not a tree then $G$ contains at least one cycle. Let $(i, j)$ be an edge on a cycle of $G$. Now assign a nonzero scaler weight $w$ to the edge $(i, j)$ and for other edges assign the weight 1 . By matrix tree theorem (weighted version), any cofactor of $L$ is the sum of the weights of all spanning trees of $G$. Note that in this case weight of a tree means the product of all edge weights of the tree. Then it is clear that any cofactor of $L$ is equal to the form $a w+b$, where $a$ is the number of spanning trees which contain the edge $(i, j)$ and $b$ is the number of spanning trees which does not contain the edge $(i, j)$. As $(i, j)$ is not a cut edge, both $a, b$

are nonzero. Now we consider $w=-\frac{b}{a}$, which is a non zero negative number. Then each cofactor of $L$ is becomes zero, so that each minor of order $n-1$ becomes zero. Hence in this case rank of $L$ is less than $(n-1)$.

We illustrate the above theorem by the following example.

Example 2.2. Consider the weighted graph $G$ (not a tree) in Figure 2. Here in the edge $(1,3)$ we assign weight $w$ and all other edges assign the weight 1 . Then the corresponding Laplacian matrix is given by

$$
L=\left[\begin{array}{cccc}
w+2 & -1 & -w & -1 \\
-1 & 2 & -1 & 0 \\
-w & -1 & 2+w & -1 \\
-1 & 0 & -1 & 2
\end{array}\right]
$$

Now $T_{1}, T_{2}, T_{3}$ and $T_{4}$ are the spanning trees of $G$ which contain the edge $(1,3)$ and $T_{5}, T_{6}, T_{7}$ and $T_{8}$ are the spanning trees of $G$ which does not contain the edge $(1,3)$. The weight of each of the tree $T_{1}, T_{2}, T_{3}$ and $T_{4}$ is $w$ and weight of each of the tree $T_{5}, T_{6}, T_{7}$ and $T_{8}$ is 1 . Sum of the weights of all spanning trees of $G$ is $4 w+4$. One can verify that each cofactor of $L$ is also $4 w+4$. Now if we take $w=-1$, then rank of $L$ is $2(<n-1)$.

Now using Theorem 2.2 and Theorem 2.3, we can characterize the trees.

Theorem 2.4. let $G$ be a graph on $n$ vertices. Then for all choices of nonsingular weight matrices of the edges, rank of the associated weighted Laplacian matrix $L$ is $(n-1) s$ (where $s$ is the size of the weight matrices) if and only if $G$ is a tree.

\section{Inverse of distance matrices of trees with matrix weights}

In this section first we state the formula for the determinant of the distance matrix of a tree with matrix weight which is obtained in 3 . 


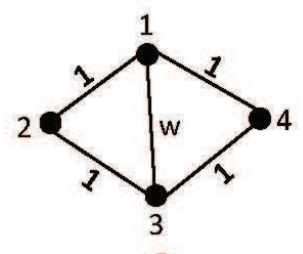

G
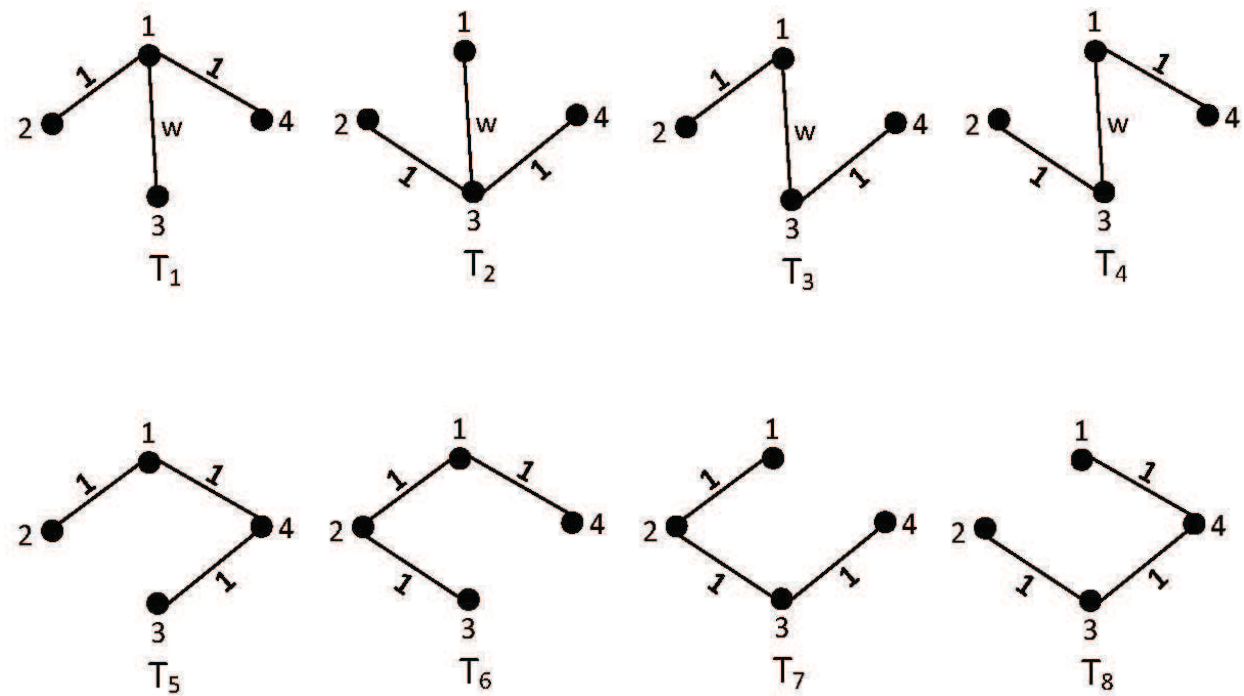

Figure 2: Graph $G$ and its spanning trees

Theorem 3.1. Let $T$ be a tree with $n$ vertices and let $W_{i}$ be the $s \times s$ edge weight matrix associated with the edge $e_{i}, i=1,2, \ldots, n-1$, and let $D$ be the distance matrix of $T$. Then

$$
\operatorname{det} D=(-1)^{(n-1) s} 2^{(n-2) s} \operatorname{det}\left(\prod_{i=1}^{n-1} W_{i}\right) \operatorname{det}\left(\sum_{i=1}^{n-1} W_{i}\right) \text {. }
$$

Theorem 3.2. Let $T$ be a tree with $n$ vertices and let $W_{i}$ be the $s \times s$ edge weight matrices for $i=1,2, \ldots, n-1$, and let $D$ be the distance matrix of $T$. Then $D$ is invertible if and only if the weight matrices $W_{i}, i=1,2, \ldots, n-1$, and $\left(\sum_{i=1}^{n-1} W_{i}\right)$ are invertible. Let $D$ be invertible and let $L$ denote the Laplacian matrix for weighting of $T$ in which each edge weight matrix is replaced by its inverse. For $i=1,2, \ldots, n$, set $\delta_{i}=2-d_{i}$, where $d_{i}$ is the degree of the vertex $i$, and $\delta^{T}=\left[\delta_{1}, \delta_{2}, \ldots, \delta_{n}\right]$. Then the inverse of $D$ is given by

$$
D^{-1}=-\frac{1}{2} L+\frac{1}{2} \delta \delta^{T} \otimes\left(\sum_{i=1}^{n-1} W_{i}\right)^{-1} .
$$

Proof. From Theorem 3.1, it is easily verifiable that $D$ is invertible if and only if $W_{i}, i=1,2, \ldots, n-1$, and $\left(\sum_{i=1}^{n-1} W_{i}\right)$ are invertible.

For the second part, we use induction on the number of vertices $n$ of the tree $T$. For $n=2, T$ is just an edge. Then 


$$
\begin{gathered}
D=\left[\begin{array}{cc}
0 & W_{1} \\
W_{1} & 0
\end{array}\right], L=\left[\begin{array}{cc}
W_{1}^{-1} & -W_{1}^{-1} \\
-W_{1}^{-1} & W_{1}^{-1}
\end{array}\right] \text { and } \delta=\left[\begin{array}{l}
1 \\
1
\end{array}\right] . \text { Then } \\
-\frac{1}{2} L+\frac{1}{2} \delta \delta^{T} \otimes W_{1}^{-1}=-\frac{1}{2}\left[\begin{array}{cc}
W_{1}^{-1} & -W_{1}^{-1} \\
-W_{1}^{-1} & W_{1}^{-1}
\end{array}\right]+\frac{1}{2}\left[\begin{array}{cc}
W_{1}^{-1} & W_{1}^{-1} \\
W_{1}^{-1} & W_{1}^{-1}
\end{array}\right] \\
=\left[\begin{array}{cc}
0 & W_{1}^{-1} \\
W_{1}^{-1} & 0
\end{array}\right]=D^{-1} .
\end{gathered}
$$

So for $n=2$, the proof is complete. For $n \geq 3$, we assume that the inverse formula holds for trees of order $n$. Let $T^{*}$ be a weighted tree on $n+1$ vertices, say $1,2, \ldots, n+1$, and the edge weights $W_{i}, i=1,2, \ldots, n$ are such that the weight matrices and $\left(\sum_{i=1}^{n} W_{i}\right)$ are invertible. We consider a pendent vertex of $T^{*}$ and index it as $n+1$ and the vertex adjacent to $n+1$ is indexed by $n$. Also let the weight of the edge with end vertices $n$ and $n+1$ be $W_{n}$. We form a new weighted tree $T$ by deleting the vertex $n+1$. We first assume that $\left(\sum_{i=1}^{n-1} W_{i}\right)$ is invertible. Then by the induction hypothesis, inverse formula for $D^{-1}$ is true for the tree $T$. Let $D, L$, and $\delta$ be the corresponding quantities for $T$ and $D^{*}, L^{*}$, and $\delta^{*}$ for $T^{*}$. Let $e_{n}, 1$ and $I$ denote the standard $n^{\text {th }}$ unit basis vector, the column vector with all entries are equal to 1 and the identity matrix of appropriate size, respectively. Then, we have

$$
L^{*}=\left[\begin{array}{c|c}
L+e_{n} e_{n}^{T} \otimes W_{n}^{-1} & -e_{n} \otimes W_{n}^{-1} \\
\hline-e_{n}^{T} \otimes W_{n}^{-1} & W_{n}^{-1}
\end{array}\right], \delta^{*}=\left[\begin{array}{c}
\delta-e_{n} \\
\hline 1
\end{array}\right]
$$

and

$$
D^{*}=\left[\begin{array}{c|c}
D & D\left(e_{n} \otimes I\right)+\mathbf{1} \otimes W_{n} \\
\hline\left(e_{n}^{T} \otimes I\right) D+\mathbf{1}^{T} \otimes W_{n} & \mathbf{0}
\end{array}\right]
$$

We assume that $H_{n-1}=\sum_{i=1}^{n-1} W_{i}$ and $H_{n}=\sum_{i=1}^{n} W_{i}$. Then

$$
\begin{aligned}
-\frac{1}{2} L^{*}+\frac{1}{2} \delta^{*} \delta^{* T} \otimes\left(\sum_{i=1}^{n} W_{i}\right)^{-1} & =-\frac{1}{2} L^{*}+\frac{1}{2} \delta^{*} \delta^{* T} \otimes H_{n}^{-1} \\
& =\left[\begin{array}{c|c}
P & Q \\
\hline R & S
\end{array}\right]
\end{aligned}
$$

where $P, Q, R$, and $S$ are given by

$$
\begin{aligned}
P & =-\frac{1}{2} L-\frac{1}{2} e_{n} e_{n}^{T} \otimes W_{n}^{-1}+\frac{1}{2}\left(\delta \delta^{T}-e_{n} \delta^{T}-\delta e_{n}^{T}+e_{n} e_{n}^{T}\right) \otimes H_{n}^{-1} \\
Q & =\frac{1}{2} e_{n} \otimes W_{n}^{-1}+\frac{1}{2}\left(\delta-e_{n}\right) \otimes H_{n}^{-1} \\
R & =\frac{1}{2} e_{n}^{T} \otimes W_{n}^{-1}+\frac{1}{2}\left(\delta^{T}-e_{n}^{T}\right) \otimes H_{n}^{-1} \\
S & =-\frac{1}{2} W_{n}^{-1}+\frac{1}{2} H_{n}^{-1}
\end{aligned}
$$

Now one can verify that

$$
\begin{array}{r}
{\left[\begin{array}{c|c}
I_{n} \otimes I_{s} & \mathbf{0} \\
\hline-e_{n}^{T} \otimes I_{s} & I_{s}
\end{array}\right]\left[\begin{array}{c|c}
D & D\left(e_{n} \otimes I\right)+\mathbf{1} \otimes W_{n} \\
\hline\left(e_{n}^{T} \otimes I\right) D+\mathbf{1}^{T} \otimes W_{n} & \mathbf{0}
\end{array}\right]\left[\begin{array}{c|c}
I_{n} \otimes I_{s} & -e_{n} \otimes I_{s} \\
\hline \mathbf{0} & I_{s}
\end{array}\right]} \\
=\left[\begin{array}{c|c}
D & \mathbf{1} \otimes W_{n} \\
\hline \mathbf{1}^{T} \otimes W_{n} & -2 W_{n}
\end{array}\right],
\end{array}
$$


and this implies

$$
\begin{aligned}
& D^{*-1}=\left[\begin{array}{c|c}
D & D\left(e_{n} \otimes I\right)+\mathbf{1} \otimes W_{n} \\
\hline\left(e_{n}^{T} \otimes I\right) D+\mathbf{1}^{T} \otimes W_{n} & \mathbf{0}
\end{array}\right]^{-1}
\end{aligned}
$$

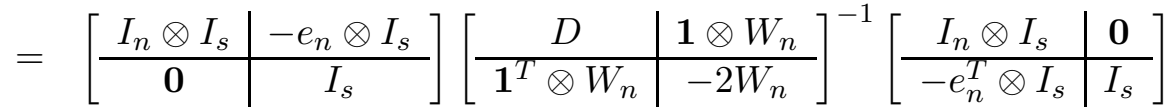

In the partitioned matrix $\left[\begin{array}{c|c}D & \mathbf{1} \otimes W_{n} \\ \hline \mathbf{1}^{T} \otimes W_{n} & -2 W_{n}\end{array}\right]$, the Schur complement of the block $D$ is given by $-2 W_{n}-\left(\mathbf{1}^{T} \otimes W_{n}\right) D^{-1}\left(\mathbf{1} \otimes W_{n}\right)=-2 W_{n}-\left(\mathbf{1}^{T} \otimes W_{n}\right)\left(-\frac{1}{2} L+\frac{1}{2} \delta \delta^{T} \otimes H_{n-1}^{-1}\right)\left(\mathbf{1} \otimes W_{n}\right)$

[By induction hypothesis]

$$
\begin{aligned}
& =-2 W_{n}-\left(\mathbf{1}^{T} \otimes W_{n}\right)\left[-\frac{1}{2} L\left(\mathbf{1} \otimes W_{n}\right)+\frac{1}{2}\left(\delta \delta^{T} \otimes H_{n-1}^{-1}\right)\left(\mathbf{1} \otimes W_{n}\right)\right] \\
& =-2 W_{n}-\left(\mathbf{1}^{T} \otimes W_{n}\right)\left[\mathbf{0}+\frac{1}{2}\left(\delta \delta^{T} \mathbf{1}\right) \otimes\left(H_{n-1}^{-1} W_{n}\right)\right]
\end{aligned}
$$

[As each block row sum of $L$ is zero]

$$
\begin{aligned}
& =-2 W_{n}-\left(\mathbf{1}^{T} \otimes W_{n}\right)\left[\delta \otimes\left(H_{n-1}^{-1} W_{n}\right)\right] \\
& \qquad\left[\text { As } \delta^{T} \mathbf{1}=\mathbf{1}^{T} \delta=2\right] \\
& =-2 W_{n}-\left(\mathbf{1}^{T} \delta\right) \otimes\left(W_{n} H_{n-1}^{-1} W_{n}\right) \\
& =-2 W_{n}-2 W_{n} H_{n-1}^{-1} W_{n}
\end{aligned}
$$

Now using a well known formula for the inverse of a partition matrix we get

$$
\left[\begin{array}{c|c}
D & \mathbf{1} \otimes W_{n} \\
\hline \mathbf{1}^{T} \otimes W_{n} & -2 W_{n}
\end{array}\right]^{-1}=\left[\begin{array}{c|c}
P_{0} & Q_{0} \\
\hline R_{0} & S_{0}
\end{array}\right]
$$

where $P_{0}, Q_{0}, R_{0}$, and $S_{0}$ are given by

$$
\begin{aligned}
P_{0} & =D^{-1}+D^{-1}\left(\mathbf{1} \otimes W_{n}\right)\left(-2 W_{n}-2 W_{n} H_{n-1}^{-1} W_{n}\right)^{-1}\left(\mathbf{1}^{T} \otimes W_{n}\right) D^{-1} \\
Q_{0} & =-D^{-1}\left(\mathbf{1} \otimes W_{n}\right)\left(-2 W_{n}-2 W_{n} H_{n-1}^{-1} W_{n}\right)^{-1} \\
R_{0} & =-\left(-2 W_{n}-2 W_{n} H_{n-1}^{-1} W_{n}\right)^{-1}\left(\mathbf{1}^{T} \otimes W_{n}\right) D^{-1} \\
S_{0} & =\left(-2 W_{n}-2 W_{n} H_{n-1}^{-1} W_{n}\right)^{-1}
\end{aligned}
$$

Now we simplify $P_{0}, Q_{0}, R_{0}$, and $S_{0}$ one by one.

$$
\begin{aligned}
P_{0} & =D^{-1}+\delta \otimes\left(H_{n-1}^{-1} W_{n}\right)\left(-2 W_{n}-2 W_{n} H_{n-1}^{-1} W_{n}\right)^{-1} \delta^{T} \otimes\left(W_{n} H_{n-1}^{-1}\right) \\
& =D^{-1}+\delta \delta^{T} \otimes\left(H_{n-1}^{-1} W_{n}\right)\left(-2 W_{n}-2 W_{n} H_{n-1}^{-1} W_{n}\right)^{-1}\left(W_{n} H_{n-1}^{-1}\right) \\
& =D^{-1}-\frac{1}{2} \delta \delta^{T} \otimes\left(H_{n-1}^{-1} W_{n} H_{n}^{-1}\right) \\
Q_{0} & =-\delta \otimes\left(H_{n-1}^{-1} W_{n}\right)\left(-2 W_{n}-2 W_{n} H_{n-1}^{-1} W_{n}\right)^{-1}=\frac{1}{2} \delta \otimes H_{n}^{-1} \text { and } R_{0}=\frac{1}{2} \delta^{T} \otimes H_{n}^{-1} \\
S_{0} & =\left(-2 W_{n}-2 W_{n} H_{n-1}^{-1} W_{n}\right)^{-1}=-\frac{1}{2}\left(W_{n}^{-1}-H_{n}^{-1}\right)
\end{aligned}
$$




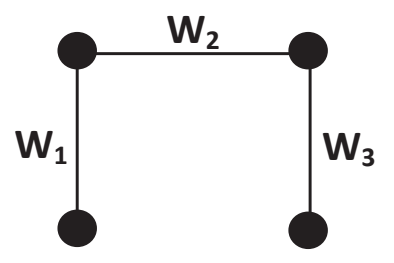

Figure 3: Path of order 4

Thus using (21) and (3) we get

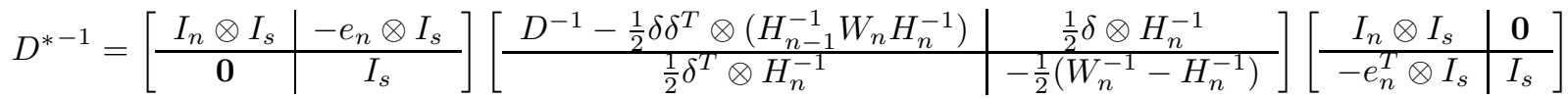

$$
\begin{aligned}
& =\left[\begin{array}{c|c}
D^{-1}-\frac{1}{2} \delta \delta^{T} \otimes\left(H_{n-1}^{-1} W_{n} H_{n}^{-1}\right)-\frac{1}{2} e_{n} \delta^{T} \otimes H_{n}^{-1} & \frac{1}{2} \delta \otimes H_{n}^{-1}+\frac{1}{2} e_{n} \otimes\left(W_{n}^{-1}-H_{n}^{-1}\right) \\
\hline \frac{1}{2} \delta^{T} \otimes H_{n}^{-1} & -\frac{1}{2}\left(W_{n}^{-1}-H_{n}^{-1}\right)
\end{array}\right]\left[\begin{array}{c|c}
I_{n} \otimes I_{s} & \mathbf{0} \\
\hline-e_{n}^{T} \otimes I_{s} & I_{s}
\end{array}\right] \\
& =\left[\begin{array}{c|c}
-\frac{1}{2} L+\frac{1}{2} \delta \delta^{T} \otimes\left(H_{n-1}^{-1}-H_{n-1}^{-1} W_{n}^{-1} H_{n}^{-1}\right)-\frac{1}{2} e_{n} \delta^{T} \otimes H_{n}^{-1} & \frac{1}{2} \delta \otimes H_{n}^{-1}+\frac{1}{2} e_{n} \otimes\left(W_{n}^{-1}-H_{n}^{-1}\right) \\
\hline \frac{1}{2} \delta^{T} \otimes H_{n}^{-1} & -\frac{1}{2}\left(W_{n}^{-1}-H_{n}^{-1}\right)
\end{array}\right]\left[\begin{array}{cc|c}
I_{n} \otimes I_{s} & \mathbf{0} \\
\hline-e_{n}^{T} \otimes I_{s} & I_{s}
\end{array}\right] \\
& =\left[\begin{array}{c|c}
-\frac{1}{2} L+\frac{1}{2} \delta \delta^{T} \otimes H_{n}^{-1}-\frac{1}{2} e_{n} \delta^{T} \otimes H_{n}^{-1} & \frac{1}{2} \delta \otimes H_{n}^{-1}+\frac{1}{2} e_{n} \otimes\left(W_{n}^{-1}-H_{n}^{-1}\right) \\
\hline \frac{1}{2} \delta^{T} \otimes H_{n}^{-1} & -\frac{1}{2}\left(W_{n}^{-1}-H_{n}^{-1}\right)
\end{array}\right]\left[\begin{array}{c|c}
I_{n} \otimes I_{s} & \mathbf{0} \\
\hline-e_{n}^{T} \otimes I_{s} & I_{s}
\end{array}\right] \\
& =\left[\begin{array}{l|l}
P & Q \\
\hline R & S
\end{array}\right] \\
& =-\frac{1}{2} L^{*}+\frac{1}{2} \delta^{*} \delta^{* T} \otimes H_{n}^{-1}
\end{aligned}
$$

Now suppose $H_{n-1}=\sum_{i=1}^{n-1} W_{i}$ is not invertible. We chose $\epsilon>0$ such that $W_{1}^{*}=W_{1}+\epsilon I$, $H_{n-1}^{*}=\sum_{i=1}^{n-1} W_{i}+\epsilon I$, and $H_{n}^{*}=\sum_{i=1}^{n} W_{i}+\epsilon I$ are simultaneously invertible. Then using the new weights of the tree $T^{*}$ inverse formula holds. Now by observing (11) and (4) we can see that the term $H_{n-1}^{-1}$ is omitted in both the equation. Hence by the continuity argument of matrices we can say that as $\epsilon \rightarrow 0, W_{1}^{*} \rightarrow W_{1},\left(W_{1}^{*}\right)^{-1} \rightarrow W_{1}^{-1},\left(H_{n}^{*}\right)^{-1} \rightarrow H_{n}^{-1}$ and $H_{n}^{*} \rightarrow H_{n}$. Hence our inverse formula is true for this case.

We note that the formula for inverse of the distance matrix of a weighted tree with the weights are invertible elements of a ring is given in [11]. One may derive the formula given from [11, Theorem 10]. However, we present an independent proof here. Next, let us illustrate the above theorem by an example.

Example 3.1. Consider a path of order 4 (Figure 3), where the edge weights are $W_{1}=\left[\begin{array}{ll}2 & 0 \\ 0 & 1\end{array}\right], W_{2}=$ $\left[\begin{array}{ll}0 & 2 \\ 1 & 0\end{array}\right], W_{3}=\left[\begin{array}{ll}1 & 0 \\ 0 & 2\end{array}\right]$ and then $\sum_{i=1}^{3} W_{i}=\left[\begin{array}{ll}3 & 2 \\ 1 & 3\end{array}\right]$. According to Theorem 3.2 , the distance 
matrix of the tree is invertible. In this case $\delta=[1,0,0,1]^{T}$ and distance matrix is given by

$$
D=\left[\begin{array}{cccc}
0 & & & \\
W_{1} & W_{1} & W_{1}+W_{2} & W_{1}+W_{2}+W_{3} \\
W_{1}+W_{2} & W_{2} & W_{2} & W_{2}+W_{3} \\
W_{1}+W_{2}+W_{3} & W_{2}+W_{3} & W_{3} & W_{3}
\end{array}\right]=\left[\begin{array}{cccccccc}
0 & 0 & 2 & 0 & 2 & 2 & 3 & 2 \\
0 & 0 & 0 & 1 & 1 & 1 & 1 & 3 \\
2 & 0 & 0 & 0 & 0 & 2 & 1 & 2 \\
0 & 1 & 0 & 0 & 1 & 0 & 1 & 2 \\
2 & 2 & 0 & 2 & 0 & 0 & 1 & 0 \\
1 & 1 & 1 & 0 & 0 & 0 & 0 & 2 \\
3 & 2 & 1 & 2 & 1 & 0 & 0 & 0 \\
1 & 3 & 1 & 2 & 0 & 2 & 0 & 0
\end{array}\right]
$$

Also the Laplacian matrix is given by

$$
L=\left[\begin{array}{cccc}
W_{1}^{-1} & -W_{1}^{-1} & 0 & 0 \\
-W_{1}^{-1} & W_{1}^{-1}+W_{2}^{-1} & -W_{2}^{-1} & 0 \\
0 & -W_{2}^{-1} & W_{2}^{-1}+W_{3}^{-1} & -W_{3}^{-1} \\
0 & 0 & -W_{3}^{-1} & W_{3}^{-1}
\end{array}\right]=\left[\begin{array}{cccccccc}
.5 & 0 & -.5 & 0 & 0 & 0 & 0 & 0 \\
0 & 1 & 0 & -1 & 0 & 0 & 0 & 0 \\
-.5 & 0 & .5 & 1 & 0 & -1 & 0 & 0 \\
0 & -1 & .5 & 1 & -.5 & 0 & 0 & 0 \\
0 & 0 & 0 & -1 & 1 & 1 & -1 & 0 \\
0 & 0 & -.5 & 0 & .5 & .5 & 0 & -.5 \\
0 & 0 & 0 & 0 & -1 & 0 & 1 & 0 \\
0 & 0 & 0 & 0 & 0 & -.5 & 0 & .5
\end{array}\right]
$$

Then one can verify that $D^{-1}=-\frac{1}{2} L+\frac{1}{2} \delta \delta^{T} \otimes\left(W_{1}+W_{2}+W_{3}\right)^{-1}$.

As a consequence of the above theorem, we obtain one of the main results presented in [1].

Corollary 3.1. [1, Theorem 3.7] Let $T$ be a tree with $n$ vertices and positive definite matrix weights. Suppose $D$ and $L$ are respectively the distance and Laplacian matrices of $T$. Let $\delta_{i}$ denote the degree of ith vertex of $T$ and $\tau=\left(2-\delta_{1}, 2-\delta_{2}, \cdots, 2-\delta_{n}\right)^{T}$. If $\Delta=\tau \otimes I_{s}$ and $R$ is the sum of all the weights of $T$, then

$$
D^{-1}=-\frac{1}{2} L+\frac{1}{2} \Delta R^{-1} \Delta^{T}
$$

Now, we present extensions of some of the results presented in [5]. Among these results, the first four parts are true for trees with nonsingular matrix weights and the last part holds true for trees with positive definite matrix weights.

Theorem 3.3. Let $T$ be a tree with $n$ vertices, let $W_{i}, i=1,2, \ldots, n-1$, be the $s \times s$ edge weight matrices, and let $D$ be the distance matrix of $T$. Let $D$ be invertible and let $L$ denote the Laplacian matrix for weighting of $T$ in which each edge weight matrix is replaced by its inverse. For $i=1,2, \ldots, n$, set $\delta_{i}=2-d_{i}$, where $d_{i}$ is the degree of the vertex $i$, and $\delta^{T}=\left[\delta_{1}, \delta_{2}, \ldots, \delta_{n}\right]$. Let $J$ denote the matrix 
of appropriate size with all entries equals to 1 . Then the following hold:

(i) $L D=\delta \mathbf{1}^{T} \otimes I_{s}-2 I_{n} \otimes I_{s}$

(ii) $\quad D L=\mathbf{1} \delta^{T} \otimes I_{s}-2 I_{n} \otimes I_{s}$

(iii) $\quad L D L=-2 L$

(iv) $\quad\left(D^{-1}-L\right)^{-1}=\frac{1}{3} D+\frac{1}{3} J \otimes \sum_{i=1}^{n-1} W_{i}$

(v) If all the weight matrices are positive definite then $Q^{T} D Q=-2 I_{(n-1) s \times(n-1) s}$, where $Q$ denotes the incidence matrix of the weighted tree $T$ by replacing each edge weight matrix by its inverse.

Proof. (i) As $D$ is invertible, so the matrices $W_{i}, i=1,2, \ldots, n-1$, and $\sum_{i=1}^{n-1} W_{i}$ are invertible and

$$
\begin{aligned}
D^{-1} & =-\frac{1}{2} L+\frac{1}{2} \delta \delta^{T} \otimes\left(\sum_{i=1}^{n-1} W_{i}\right)^{-1} \\
\Rightarrow I_{n s} & =-\frac{1}{2} L D+\frac{1}{2}\left[\delta \delta^{T} \otimes\left(\sum_{i=1}^{n-1} W_{i}\right)^{-1}\right] D \\
\Rightarrow L D & =\left[\delta \delta^{T} \otimes\left(\sum_{i=1}^{n-1} W_{i}\right)^{-1}\right] D-2 I_{n s}
\end{aligned}
$$

Again we have

$$
\begin{aligned}
\left(\mathbf{1}^{T} \otimes I_{s}\right) D^{-1} & =\left(\mathbf{1}^{T} \otimes I_{s}\right)\left[-\frac{1}{2} L+\frac{1}{2} \delta \delta^{T} \otimes\left(\sum_{i=1}^{n-1} W_{i}\right)^{-1}\right] \\
& =0+\frac{1}{2} \mathbf{1}^{T} \delta \delta^{T} \otimes\left(\sum_{i=1}^{n-1} W_{i}\right)^{-1} \\
& =\delta^{T} \otimes\left(\sum_{i=1}^{n-1} W_{i}\right)^{-1} \\
\Rightarrow\left(\mathbf{1}^{T} \otimes I_{s}\right) & =\left[\delta^{T} \otimes\left(\sum_{i=1}^{n-1} W_{i}\right)^{-1}\right] D \\
\Rightarrow\left(\delta \otimes I_{s}\right)\left(\mathbf{1}^{T} \otimes I_{s}\right) & =\left[\delta \delta^{T} \otimes\left(\sum_{i=1}^{n-1} W_{i}\right)^{-1}\right] D \\
\Rightarrow\left[\delta \delta^{T} \otimes\left(\sum_{i=1}^{n-1} W_{i}\right)^{-1}\right] D & =\left(\delta \mathbf{1}^{T} \otimes I_{s}\right)
\end{aligned}
$$

Hence from (5) we get

$$
L D=\delta \mathbf{1}^{T} \otimes I_{s}-2 I_{n} \otimes I_{s}
$$

(ii) Applying similar technique as in (i) we have the following,

$D L=D\left[\delta \delta^{T} \otimes\left(\sum_{i=1}^{n-1} W_{i}\right)^{-1}\right]-2 I_{n s}, D^{-1}\left(\mathbf{1} \otimes I_{s}\right)=\delta \otimes\left(\sum_{i=1}^{n-1} W_{i}\right)^{-1}$ and $\mathbf{1} \delta^{T} \otimes I_{s}=D\left[\delta \delta^{T} \otimes\left(\sum_{i=1}^{n-1} W_{i}\right)^{-1}\right]$ 
Hence using these we get that

$$
D L=\mathbf{1} \delta^{T} \otimes I_{s}-2 I_{n} \otimes I_{s}
$$

(iii)

$$
\begin{aligned}
L D L & =\left[\delta \mathbf{1}^{T} \otimes I_{s}-2 I_{n} \otimes I_{s}\right] L \\
& =\left[\delta \mathbf{1}^{T} \otimes I_{s}\right] L-2 L \\
& =\delta\left[\left(\mathbf{1}^{T} \otimes I_{s}\right) L\right]-2 L \\
& =0-2 L \\
& =-2 L
\end{aligned}
$$

(iv) Let us assume $\sum_{i=1}^{n-1} W_{i}=H_{n-1}$. Then

$$
\begin{aligned}
\left(D^{-1}-L\right)\left[\frac{1}{3} D+\frac{1}{3} J \otimes H_{n-1}\right] & =\frac{1}{3} D^{-1} D-\frac{1}{3} L D+\frac{1}{3} D^{-1}\left(J \otimes H_{n-1}\right)-\frac{1}{3} L\left(J \otimes H_{n-1}\right) \\
& =\frac{1}{3} I_{n s}-\frac{1}{3}\left[\delta \mathbf{1}^{T} \otimes I_{s}-2 I_{n} \otimes I_{s}\right]+\frac{1}{3} D^{-1}\left(\mathbf{1 1}^{T} \otimes H_{n-1}\right)-\frac{1}{3} L\left(\mathbf{1 1}^{T} \otimes H_{n-1}\right) \\
& =I_{n s}-\frac{1}{3} \delta \mathbf{1}^{T} \otimes I_{s}+\frac{1}{3} D^{-1}\left(\mathbf{1} \otimes I_{s}\right)\left(\mathbf{1}^{T} \otimes H_{n-1}\right)-\frac{1}{3} L\left(\mathbf{1} \otimes I_{s}\right)\left(\mathbf{1}^{T} \otimes H_{n-1}\right) \\
& =I_{n s}-\frac{1}{3} \delta \mathbf{1}^{T} \otimes I_{s}+\frac{1}{3}\left(\delta \otimes H_{n-1}^{-1}\right)\left(\mathbf{1}^{T} \otimes H_{n-1}\right)-0 \\
& =I_{n s}-\frac{1}{3} \delta \mathbf{1}^{T} \otimes I_{s}+\frac{1}{3} \delta \mathbf{1}^{T} \otimes I_{s} \\
& =I_{n s}
\end{aligned}
$$

Hence

$$
\left(D^{-1}-L\right)^{-1}=\frac{1}{3} D+\frac{1}{3} J \otimes \sum_{i=1}^{n-1} W_{i}
$$

(v) If all the weight matrices are positive definite, then the incidence matrix $Q$ is well defined and Laplacian Matrix $L=Q Q^{T}$. Since,

$$
\begin{aligned}
L D & =\delta \mathbf{1}^{T} \otimes I_{s}-2 I_{n} \otimes I_{s}, \\
\text { we have } Q Q^{T} D Q & =\left(\delta \mathbf{1}^{T} \otimes I_{s}\right) Q-2\left(I_{n} \otimes I_{s}\right) Q \\
& =\left(\delta \otimes I_{s}\right)\left(\mathbf{1}^{T} \otimes I_{s}\right) Q-2 Q \\
& =0-2 Q \\
& =-2 Q
\end{aligned}
$$

Now as all the weight matrices are nonsingular, the incidence matrix $Q$ has full column rank and thus it admits a left inverse. Hence

$$
Q^{T} D Q=-2 I_{(n-1) s \times(n-1) s}
$$


Remark 3.1. Part(iii) of the above theorem gives an alternate simple proof for a more general version of Lemma 3.2. of [1]. The proof presented in [1] uses mathematical induction.

A matrix $G$ of order $n \times m$ is said to be a generalized inverse (or a $g$-inverse) of $A$ if $A G A=A$. Next, we shall consider the properties related to distance matrix, Laplacian matrix and g-inverse. Let $e_{i j}$ be a $n \times 1$ vector with $i$ coordinate equal to $1, j$ coordinate equal to -1 , and zeros elsewhere. Let $B$ be an $n s \times n s$ matrix which is partitioned into the form

$$
B=\left(\begin{array}{cccc}
B_{1,1} & B_{1,2} & \cdots & B_{1, n} \\
B_{2,1} & B_{2,2} & \cdots & B_{2, n} \\
\cdots & \cdots & \cdots & \cdots \\
B_{n, 1} & B_{n, 2} & \cdots & B_{n, n}
\end{array}\right)
$$

where each $B_{i, j}$, for $i, j=1,2, \cdots, n$, is a submatrix of order $s$. Then $\left(e_{i j} \otimes I_{s}\right)^{T} B\left(e_{i j} \otimes I_{s}\right)=$ $B_{i, i}+B_{j, j}-B_{i, j}-B_{j, i}$.

Theorem 3.4. Let $G$ be a weighted graph on $n$ vertices, where each weight is a positive definite matrix. Let $D$ be the distance matrix of $G$ and $L$ denote the Laplacian matrix of $G$ in which each edge weight matrix is replaced by its inverse.

(i)If $H^{1}$ and $H^{2}$ are any two g-inverse of $L$, then $\left(e_{i j} \otimes I_{s}\right)^{T} H^{1}\left(e_{i j} \otimes I_{s}\right)=\left(e_{i j} \otimes I_{s}\right)^{T} H^{2}\left(e_{i j} \otimes I_{s}\right)$.

(ii) If $G$ is a tree on $n$ vertices and $H$ is a g-inverse of $L$, then $H_{i, i}+H_{j, j}-H_{i, j}-H_{j, i}=D_{i, j}$.

Proof. (i) We have $\operatorname{Rank}(L)=\operatorname{Rank}\left(Q Q^{T}\right)=\operatorname{Rank}(Q)=n s-s$. Also the vectors $1 \otimes e_{i}$ for $i=$ $1,2, \cdots, s$ form a basis for the null space of $L$. Since $\left(\mathbf{1} \otimes e_{k}\right)^{T}\left(e_{i, j} \otimes I_{s}\right)=0$ for all $i, j, k \in\{1,2, \cdots, n\}$. Thus column space of $e_{i, j} \otimes I_{s}$ is a subspace of column space of $L$. So there exist a matrix $C$ such that $e_{i, j} \otimes I_{s}=L C$. Then

$$
\left(e_{i j} \otimes I_{s}\right)^{T}\left(H^{1}-H^{2}\right)\left(e_{i j} \otimes I_{s}\right)=C^{T} L^{T}\left(H^{1}-H^{2}\right) L C=C^{T}\left(L H^{1} L-L H^{2} L\right) C=0
$$

Hence $\left(e_{i j} \otimes I_{s}\right)^{T} H^{1}\left(e_{i j} \otimes I_{s}\right)=\left(e_{i j} \otimes I_{s}\right)^{T} H^{2}\left(e_{i j} \otimes I_{s}\right)$.

(ii) From Theorem 3.3, we have $L D L=-2 L$. Thus $-\frac{D}{2}$ is a $g$-inverse of $L$. Then by part (i) $\left(e_{i j} \otimes I_{s}\right)^{T} H\left(e_{i j} \otimes I_{s}\right)=\left(e_{i j} \otimes I_{s}\right)^{T}\left(-\frac{D}{2}\right)\left(e_{i j} \otimes I_{s}\right)$.

So $H_{i, i}+H_{j, j}-H_{i, j}-H_{j, i}=-\frac{1}{2} D_{i, i}-\frac{1}{2} D_{j, j}+\frac{1}{2} D_{i, j}+\frac{1}{2} D_{j, i}=D_{i, j}$

Next let us recall a known result from [3].

Theorem 3.5. 3] Let $T$ be a tree with $n$ vertices, let $W_{i}$ be a positive definite $s \times s$ edge weight matrix associated with the edge $e_{i}, i=1,2, \cdots, n-1$, and let $D$ be the distance matrix of $T$. Then $D$ has $s$ positive and $(n-1) s$ negative eigenvalues.

Our next result gives some interlacing inequality for the eigenvalues of $D$ and $L$ where the graph is a weighted tree on $n$ vertices. This result extends a result given in [9]. 
Theorem 3.6. Let $T$ be a weighted tree on $n$ vertices, where each weight is a positive definite matrix of order s. Let $D$ be the distance matrix of $T$ and $L$ denote the Laplacian matrix of $T$ in which each edge weight matrix is replaced by its inverse. Let $\mu_{1} \geq \mu_{2} \geq \cdots \geq \mu_{s}>0>\mu_{s+1} \geq \cdots \geq \mu_{n s}$ be the eigenvalues of $D$ and $\lambda_{1} \geq \lambda_{2} \geq \cdots \geq \lambda_{n s-s}>\lambda_{n s-s+1}=\cdots=\lambda_{n s}=0$ be the eigenvalues of $L$. Then

$$
\mu_{s+i} \leq-\frac{2}{\lambda_{i}} \leq \mu_{i} \text { for } i=1,2, \cdots, n s-s
$$

Proof. Let $Q$ be the $n s \times(n-1) s$ vertex-edge incidence matrix in which each edge weight matrix is replaced by its inverse. As $Q$ has rank $(n-1) s$, columns of $Q$ are linearly independent. By performing Gram-Schmidt process on these columns of $Q$, we can say that there exist an $(n-1) s \times(n-1) s$ nonsingular matrix $M$ such that the columns of $Q M$ are orthonormal. As we have $\left(\mathbf{1}^{T} \otimes I_{s}\right) Q=0$, $U=\left[Q M, \frac{1}{\sqrt{n}}\left(\mathbf{1} \otimes I_{s}\right)\right]$ is an orthogonal matrix and so the eigenvalues of $D$ and $U^{T} D U$ are equal. Now

$$
\begin{aligned}
U^{T} D U & =\left[\begin{array}{cc}
M^{T} Q^{T} D Q M & \frac{1}{\sqrt{n}} M^{T} Q^{T} D\left(\mathbf{1} \otimes I_{s}\right) \\
\frac{1}{\sqrt{n}}\left(\mathbf{1}^{T} \otimes I_{s}\right) D Q M & \frac{1}{n}\left(\mathbf{1}^{T} \otimes I_{s}\right) D\left(\mathbf{1} \otimes I_{s}\right)
\end{array}\right] \\
& =\left[\begin{array}{cc}
-2 M^{T} M & \frac{1}{\sqrt{n}} M^{T} Q^{T} D\left(\mathbf{1} \otimes I_{s}\right) \\
\frac{1}{\sqrt{n}}\left(\mathbf{1}^{T} \otimes I_{s}\right) D Q M & \frac{1}{n}\left(\mathbf{1}^{T} \otimes I_{s}\right) D\left(\mathbf{1} \otimes I_{s}\right)
\end{array}\right] \quad \text { [By Theorem 3.3. Part (v)] }
\end{aligned}
$$

Assume that $K=Q^{T} Q$, then $K$ is nonsingular. Also $M^{T} K M=M^{T} Q^{T} Q M=I$ implies $K^{-1}=$ $M M^{T}$. As $M$ is nonsingular, $K^{-1}$ and $M^{T} M$ have the same eigenvalues. Again the nonzero eigenvalues of $L=Q Q^{T}$ are the eigenvalues of $K=Q^{T} Q$. Thus $-\frac{2}{\lambda_{1}} \geq-\frac{2}{\lambda_{2}} \geq \cdots \geq-\frac{2}{\lambda_{(n-1) s}}$ are the eigenvalues of $-2 K^{-1}$ as well as $-2 M^{T} M$. Hence by interlacing theorem we have

$$
\mu_{s+i} \leq-\frac{2}{\lambda_{i}} \leq \mu_{i} \text { for } i=1,2, \cdots, n s-s .
$$

Our next result, which is immediate from the previous theorem, gives an interlacing inequality for scaler weighted trees.

Corollary 3.2. Let $T$ be a weighted tree on $n$ vertices with each weights are positive number. Let $D$ be the distance matrix of $T$ and $L$ be the Laplacian matrix of $T$ that arise by replacing each weight by its reciprocal. Let $\mu_{1}>0>\mu_{2} \geq \cdots \geq \mu_{n}$ be the eigenvalues of $D$ and $\lambda_{1} \geq \lambda_{2} \geq \cdots \geq \lambda_{n-1}>\lambda_{n}=0$ be the eigenvalues of $L$. Then

$$
0>-\frac{2}{\lambda_{1}} \geq \mu_{2} \geq-\frac{2}{\lambda_{2}} \geq \mu_{3} \geq \cdots \geq-\frac{2}{\lambda_{n-1}} \geq \mu_{n} .
$$

Acknowledgement: M. Rajesh Kannan would like to thank Department of Science and Technology for the financial support. 


\section{References}

[1] R. Balaji and R. B. Bapat, Block distance matrices, Electron. J. Linear Algebra 16 (2007), 435443. MR 2365897

[2] R. Bapat, S. J. Kirkland, and M. Neumann, On distance matrices and Laplacians, Linear Algebra Appl. 401 (2005), 193-209. MR 2133282

[3] R. B. Bapat, Determinant of the distance matrix of a tree with matrix weights, Linear Algebra Appl. 416 (2006), no. 1, 2-7. MR 2232916

[4] R. B. Bapat, A. K. Lal, and Sukanta Pati, A q-analogue of the distance matrix of a tree, Linear Algebra Appl. 416 (2006), no. 2-3, 799-814. MR 2242465

[5] Ravindra B. Bapat, Graphs and matrices, second ed., Universitext, Springer, London; Hindustan Book Agency, New Delhi, 2014. MR 3289036

[6] R. L. Graham and L. Lovász, Distance matrix polynomials of trees, Adv. in Math. 29 (1978), no. 1, 60-88. MR 0480119

[7] R. L. Graham and H. O. Pollak, On the addressing problem for loop switching, Bell System Tech. J. 50 (1971), 2495-2519. MR 0289210

[8] Roger A. Horn and Charles R. Johnson, Topics in matrix analysis, Cambridge University Press, Cambridge, 1994, Corrected reprint of the 1991 original. MR 1288752

[9] Russell Merris, The distance spectrum of a tree, J. Graph Theory 14 (1990), no. 3, 365-369. MR 1060864

[10] Hui Zhou, The inverse of the distance matrix of a distance well-defined graph, Linear Algebra Appl. 517 (2017), 11-29. MR 3592007

[11] Hui Zhou and Qi Ding, The distance matrix of a tree with weights on its arcs, Linear Algebra Appl. 511 (2016), 365-377. MR 3557839 